\title{
A model for setting up development programs for logistics systems in the electric power industry to achieve electric power security
}

\author{
Elizaveta Zotova ${ }^{1}$, Vladimir Anisimov ${ }^{1}$, Evgenii Anisimov ${ }^{2}$, Daria Melnik $^{3}$, and Anatoly \\ Chernysh ${ }^{4, *}$ \\ ${ }^{1}$ Peter the Great St. Petersburg Polytechnic University (SPbPU), Graduate School of Business and \\ Management, 29, Polytechnicheskaya Str., 195251, St. Petersburg, Russia \\ ${ }^{2}$ People's Friendship University of Russia (RUDN University), 6, Miklukho-Maklaya Str., 117198, \\ Moscow, Russia \\ ${ }^{3}$ Eurasian Economic Commission, 2 Letnikovskaya Str., 115114, Moscow, Russia \\ ${ }^{4}$ Concern Radio-Technical and Information Systems, 10, March 8 Str., 127083, Moscow, Russia
}

\begin{abstract}
The electric power security of the Russian Federation is one of the most important factors influencing the economy and social sphere, as well as the living conditions and activities of Russian citizens. Thus, one of the global issues concerning electric power security is to keep a balance between the demand for resources and the lack of ability of the corresponding logistic systems to provide continuity and synchronize the processes of production, distribution and delivery of enough electric power to consumers. The urgent need for purposeful development of logistics systems in the electric power industry and to ensure electric power security determines the relevance of improving the methodological tools for taking rational decisions in planning and managing this process. This paper is aimed at improving these tools. It proposes a generalized structure of the model and an algorithm for solving the problem related to forming the best variant of the logistics system development program in the electric power industry. The minimization of financial costs is used as a criterion of optimality in the model. To take into account the uncertainty inherent in the development of the logistics system in the electric power industry, the it is necessary to set the intervals of the possible costs of the project components. To resolve this problem an iterative algorithm is proposed so that an expedient variant of the project is formed consistently.
\end{abstract}

\section{Introduction}

One of the most important conditions for a high standard of living and activity of modern society is electricity. Therefore, the electric power industry, which generates, transmits and sells electricity, plays a special role in the development of the economy and the social sphere [1]. A lot of companies operate in this economic sector in the Russian Federation. At the same time, the stability and competitiveness of these companies today,

\footnotetext{
${ }^{*}$ Corresponding author: an-33@yandex.ru
} 
and, consequently, the electric power security of the state, largely depends on the ability of their logistics systems to effectively coordinate the processes of generating, distributing and supplying enough electricity to the consumers [2 - 6].

In Russia the problems of such coordination is aggravated by the uneven territorial distribution of electricity generating companies across the regions of the country and the weak electricity connections between the European part of the country and the regions of the Arctic, Siberia and the Far East. Thus, developing the logistics systems in the electric power industry is one of the strategic objectives for energy security. The task is complex and time-consuming. The decisions taken in this area have to be well-thought to avoid material and reputational costs associated with possible miscalculations as the development of electric power logistics systems is planned at the macro and micro levels [7 - 12]. The purpose of this article is to build a mathematical model and an algorithm for elaborating the logistics system development program in the electric power industry so that electric power security can be ensured.

\section{Materials and Methods}

The proposed model is aimed at forming a development program for the logistics system in the electric power industry provided that the development goals of the program are clearly formulated, and there is a certain set of partial projects, and each of them is able to ensure the achievement of a certain subset of these goals. The best variant of the program is a set of partial projects where all the objectives of electric power security are reached with minimal financial costs. The methods of solving such problems are based on the tools of mathematical programming theory [13 - 17]. When building a mathematical optimization model, it is necessary to take into account that if any of the partial projects are included in the program, a certain amount of different resources must be involved. The total amount of resources of each type is limited. The financial costs of attracting resources to various projects depend on the choice of suppliers, the time and methods of delivery, the characteristics of storage and other features of each partial project. It is also necessary to take into account the uncertainty of these costs caused by uncontrollable factors.

\section{Results}

The considered optimization problem encountered in projects aimed at developing logistic systems in the electric power industry can be formally represented as a discrete mathematical programming model of the following kind.

It is required to determine the variant

$$
X=\left\|x_{i k j}^{*}\right\|, i=\overline{1, I}, k=\overline{1, K}, j=\overline{1, J}
$$

of the logistics system development program so that:

$$
P(X)=\min _{x_{i k j}} \sum_{i=1}^{I} \sum_{k=1}^{K} \sum_{j=1}^{J} x_{i k j} \cdot p_{i k j},
$$

at:

$$
x_{i k j} \leq z_{k j}, i=\overline{1, I}, k=\overline{1, K}, j=\overline{1, J}
$$




$$
\begin{gathered}
\sum_{j=1}^{J} z_{k j} \geq 1, k=\overline{1, K} \\
\sum_{j=1}^{J} x_{i j k}=F_{i k}, i=\overline{1, I}, k=\overline{1, K} \\
\sum_{k=1}^{K} \sum_{j=1}^{J} x_{i j k} \leq F_{i}, i=\overline{1, I} \\
\sum_{k=1}^{K} \sum_{i=1}^{I} x_{i j k} \geq 1, j=\overline{1, J} \\
p_{i k j}=\frac{p_{i k j}}{z_{k j}}, i=\overline{1, I}
\end{gathered}
$$

Where

$$
\begin{gathered}
\left\{\begin{array}{l}
\int 1, \text { if } k-\text { th project can achieve } \\
j-\text { th development goal } ; \\
0, \text { in the contrary case; }
\end{array}\right. \\
x_{i k j}=\left\{\begin{array}{l}
1, \text { if resources of } i-\text { th type and project of } k-\text { th type } \\
\text { are used to achieve } j-\text { th development goal; } \\
0, \text { in the contrary case } ;
\end{array}\right.
\end{gathered}
$$

$P$ is the total cost of the logistics system development program;

$I$ is the identifier of the type of resource involved in the projects of the logistics system development program;

$k$ is the identifier of the partial project of the logistics system development;

$j$ is the identifier of the partial development goal;

$p_{i k j}$ is the financial cost of using resources of the $i$-th type in the implementation of the $k$-th partial project to achieve the $j$-th partial goal of the logistics system development;

$I$ is the number of resource types;

$K$ is the number of partial project options that can be included in the logistics system development program;

$J$ is the total number of partial goals for the logistics system development;

$F i$ is the total amount of resources of the $i$-th type, which can be used in the logistics system development program;

$F_{i k}$ is the amount of resource of the $i$-th type, used in the development program of the $k$ th project.

In this model, matrix (1) defines a variant of the composition of the partial projects and resources to achieve the objectives of the logistics system development. 
The objective function (2) establishes the requirements for minimizing financial costs. Condition (3) ensures that the possibilities of partial projects are suitable for the achievement of the development goals of the logistics system. Condition (4) determines that the set of the partial projects can ensure the achievement of all the development goals of the logistics system. It follows from condition (5) that in order to include the $k$-th project in the development program appropriate certain amount of resources of the corresponding type is required. Condition (6) means that the amount of resources of the $i$-th type necessary to achieve the development goals of the logistics system cannot exceed their total amount. Condition (7) means that the projects and resources included in the development program must ensure the achievement of all the development goals of the logistics system. According to condition (8) the possibility of including the projects that do not ensure the achievement of the corresponding development goals in the development program is eliminated.

To determine the costs $p_{i k j}$ we can use the relevant regulatory documents and experience in the implementation of similar projects in the past. It is also necessary to take into account the uncertainty of these characteristics due to the impact of uncontrollable factors. As a rule, to account for uncertainty it is advisable to represent costs in the form of fuzzy variables, defined by the intervals of their possible values [18 - 23]. It is assumed that the value of $p_{i k j}$ is between the lower and the upper limits of the interval:

$$
a_{i k j} \leq p_{i k j} \leq b_{i k j}
$$

where $a_{i k j}$ is the lower limit of the interval of possible values of $p_{i k j}$;

$b_{i k j}$ is the upper limit of the interval of possible values of $p_{i k j}$.

Within the interval $p_{i k j}$ quantities can be assumed to be random. In this case, since the distribution of the random variable $p_{i k j}$ is concentrated on the interval (9) and, as a rule, has a unimodal character, we can assume that its distribution function has the form of a betadistribution:

$$
F(\alpha, \beta)=\frac{1}{B(\alpha, \beta)} \int_{a_{i k j}}^{b_{i k j}} p_{i k j}^{\alpha-1}\left(1-p_{i k j}\right)^{\beta-1} d p_{i k j}
$$

where $\alpha, \beta$ are the beta distribution parameters;

$B(\alpha, \beta)$ is the beta function.

In this case, its mathematical expectation can be taken as an estimate of the value $p_{i k j}$. With known $a_{i k j}, b_{i k j}$ the mathematical expectation of the value $p_{i k j}$ is determined by the relation:

$$
\overline{p_{i k j}}=\frac{3 a_{i k j}+2 b_{i k j}}{5}, i=\overline{1, I} ; k=\overline{1, K} ; j=\overline{1, J},
$$

and with known $a_{i k j}, b_{i k j}, m_{i k j}$ by the ratio:

$$
\overline{p_{i k j}^{*}}=\frac{a_{i k j}+4 m_{i k j}+b_{i k j}}{6}, i=\overline{1, I} ; k=\overline{1, K} ; j=\overline{1, J},
$$

where $m_{i k j}$ is the mode of the distribution, which corresponds to the most probable value of costs and is determined on the basis of the previous experience.

Given relations (11), (12), the objective function (2) takes the form: 


$$
\bar{P}(X)=\min _{x_{i j j}} \sum_{i=1}^{I} \sum_{k=1}^{K} \sum_{j=1}^{J} x_{i k j} \overline{p_{i k j}}
$$

or

$$
\bar{P}(X)=\min _{x_{i k j}} \sum_{i=1}^{I} \sum_{k=1}^{K} \sum_{j=1}^{J} x_{i k j} \overline{p_{i k j}^{*}},
$$

where $\bar{P}(X)$ is the mathematical expectation of the total costs within the interval $[\hat{a}, \hat{b}]$.

The values $\hat{a}$ and $\hat{b}$ represent the total lower and upper limits of the distribution interval of the value $\overline{P_{T P}}(X)$, respectively:

$$
\begin{aligned}
& \hat{a}(X)=\sum_{i=1}^{I} \sum_{k=1}^{K} \sum_{j=1}^{J} a_{i j k} \cdot x_{i j j}^{*}, \\
& \hat{b}(X)=\sum_{i=1}^{I} \sum_{k=1}^{K} \sum_{j=1}^{J} b_{i j k} \cdot x_{i j j}^{*} .
\end{aligned}
$$

Since the real value of costs $P$ is random and represents the sum of random variables $p_{i k j}$, we can assume that the distribution law of $P(X)$ is normal, with the density of distribution being:

$$
f(P)=\frac{1}{\sqrt{2 \pi \bar{D}(X)}} \cdot e^{-\frac{(P(X)-\bar{P}(X))^{2}}{2 \bar{D}(X)}},
$$

where $\bar{D}$ is the variance of the total costs within the interval $[\hat{a}, \hat{b}]$.

If $a_{i k j}, b_{i k j}$ are known, the variance is calculated by the formula:

$$
\bar{D}(X)=\sum_{i=1}^{I} \sum_{k=1}^{K} \sum_{j=1}^{J} x_{i k j}^{*} D_{i k j}
$$

and with $a_{i k j}, b_{i k j}, m_{i k j}$ being known by the formula:

$$
\bar{D}(X)=\sum_{i=1}^{I} \sum_{k=1}^{K} \sum_{j=1}^{J} x_{i k j}^{*} D_{i k j}^{*},
$$

where the values of $D_{i k j}, D^{*} i k j$ are estimates of the cost variance.

In this case, the value of $D_{i k j}$ is determined by the formula:

$$
D_{i k j}=\left(\frac{b_{i k j}-a_{i k j}}{5}\right)^{2},
$$

and the value of $D^{*} i k j$ - by the formula: 


$$
D_{i k j}^{*}=\left(\frac{b_{i k j}-a_{i k j}}{6}\right)^{2}
$$

For assessing economic risks the probability that the value $P(X)$ will not exceed a given value $\left[P^{*}(X)[24,25]\right.$ is an important characteristic of the formed variant of the development program of the logistics system in the electric power industry. This characteristic is defined by the ratio:

$$
\theta=\int_{-\infty}^{P^{*}(X)} \frac{1}{\sqrt{2 \pi \bar{D}(X)}} \cdot e^{-\frac{\left(P^{*}(X)-\bar{P}(X)\right)^{2}}{2 \bar{D}(X)}}
$$

Model (1) - (8) belongs to the class of NP-complex discrete programming models [2630]. To solve the problem, it is proposed to use an iterative algorithm for the sequential formation of an expedient variant of the development program of the logistics system.

At each iteration $r$ of this algorithm, the cost-preferred project $k^{r}$ is selected and a fragment of the plan $X^{r}$ associated with this selection is formed.

To represent the algorithm, let us introduce the following notations:

$S^{r}$ is the set of partial projects included in the generated version of the program $X^{r}$ at the $r$-th iteration;

$G^{r}$ is the set of project types that can be included in the generated version of the $X^{r}$ program at the $r$-th iteration;

$E^{r}$ is the set of project types that cannot be included in the generated version of the $X^{r}$ program at the $r$-th iteration.

$H^{r}$ is the set of development goals considered at the $r$-th iteration, which require additional projects and resources.

The scheme of the algorithm is shown in Figure 1 [29].

In Block 1 the initial data are set:

- set of projects $O=\{1,2, \ldots, K\}$;

$-\operatorname{set} F=\left\{F_{i} \ldots F_{I}\right\}, i=\overline{1, I}$;

$-\operatorname{set} F_{k}=\left\{F_{i k} \ldots F_{I k}\right\}, i=\overline{1, I}, k=\overline{1, K}$;

- $\operatorname{set} H=\{1,2, \ldots, J\}$;

- matrix $Z=\left\|z_{k j}\right\|, k=\overline{1, K} ; j=\overline{1, J}$;

- financial costs (cost estimates) $p_{i k j}, i=\overline{1, I} ; k=\overline{1, K} ; j=\overline{1, J}$.

In Block 2 the sets $\mathrm{Sr},{ }^{\mathrm{Gr}},{ }^{\mathrm{Er}}, \mathrm{Hr},{ }^{\mathrm{Fr}}$ are formed. At this, we assume $r=1$,

$$
S^{r}=\varnothing ; G^{r}=O ; H^{r}=H ; G^{r}=\varnothing ; F^{r}=F
$$

In Block 3, the projects are ranked by priority. The ranking criterion is the parameter:

$$
h_{k}^{r}=\frac{\sum_{j \in H_{k}^{r}} \sum_{i=1}^{I} F_{i k} p_{i k j}}{\sum_{j \in H_{k}^{r}} z_{k j}}, k \in G^{r}
$$




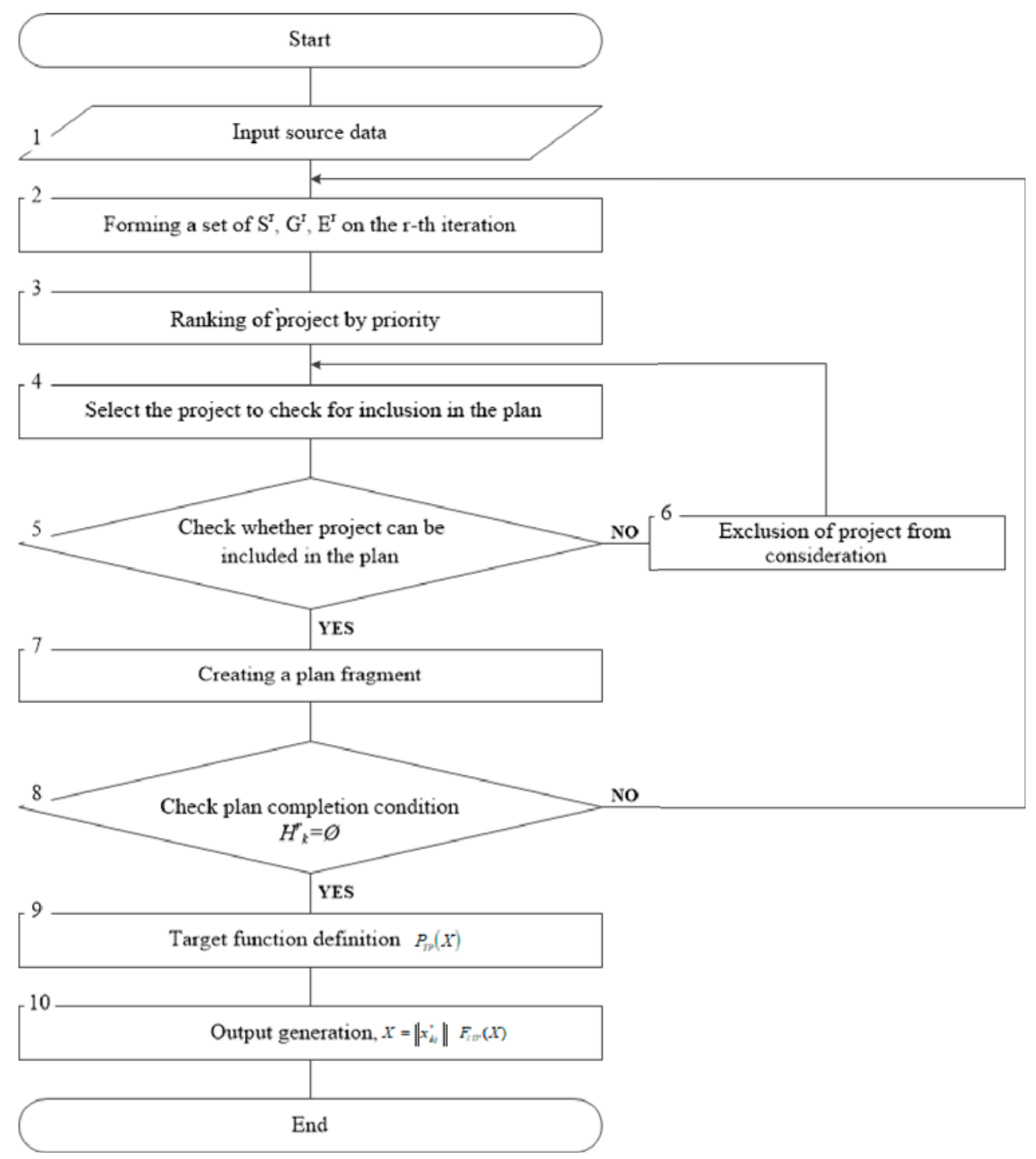

Fig. 1. Scheme of the algorithm according to which the development program of the logistics system is formed.

In Block 4, a selection of projects is made to check whether the generated variant of the $X^{r}$. program can be included. The order of selection is determined by a series of variations:

$$
h_{k l}^{r} \leq h_{k l+1}^{r} \leq h_{k l+2}^{r} \ldots \leq h_{k L}^{R},
$$

where $l$ is the serial number of the project in the variation series $(l=1,2, \ldots)$, in which case the $k$-th project with a lower order number is taken into consideration first.

In Block 5 the possibility of inclusion of the project in the formed version of the program $X^{r}$ is checked. For this purpose, the $k$-th project, accepted for consideration, is checked for compliance with conditions (5) and (6).

If these conditions are met, we proceed to step 7 . If the conditions are not met, go to step 6 . 
Block 6 modifies the sets $G^{r}$ and $E^{r}$, i.e. it is assumed that $k_{l}^{r} \notin G^{r}$ and $k_{l}^{r} \in E^{r}$.

In Block 7 the next fragment of the program is formed taking into account the selected projects, i.e. it is assumed that for all $x_{i j k}=1$ for any $i$ for which $F_{i k} \geq 0, z_{k j}=1$.

In Block 8 the condition of the end of program formation is checked.

If $H_{k}^{r}=\varnothing$, then we proceed to step 9.

If not, then go to step 2, which relies:

- iteration $r=r+1$;

- the project $k_{l}^{r-1}$ included in the fragment of the program $X^{r-1}$ formed at the previous iteration is excluded from the set $G^{r}$;

- the project $k_{l}^{r-1}$ included in $X^{r-1}$ is included in the set $S^{r}$;

- all partial development goals included in $X^{r-1}$ are excluded from the set $H^{r}$.

- the $E^{r}$ set includes the project $k_{l}^{r-1}$, which, given the assignments made earlier, cannot be included in $X$ in subsequent iterations;

- elements $F_{i}^{r}$ of the set $F^{r}$ are recalculated taking into account the resources to be used in the projects included in the variant of the program that was formed in the previous iteration

$$
\left(F_{i}^{r}=F_{i}^{r-1}-F_{k_{l}^{r-1}}, i=\overline{1, I}\right) \text {. }
$$

Block 9 defines the value of the objective function.

Block 10 generates output data: program variant $X$ and financial costs $P$.

\section{Discussion}

Electric power security, which ensures stable and uninterrupted supply and availability of electric power resources for businesses and residents, is one of the essential factors of socio-economic development of Russia's regions. At the same time, it is known from experience that the level of electric power security significantly depends on the quality of the logistics systems in the electric power industry.

Therefore, justifying effective programs for the development of these systems aimed at ensuring electric power security is really important. The complexity of the solution to this problem necessitates the development of the appropriate scientific and methodological tools for comprehensive justification of these programs. The methodological approach that is proposed in the paper for building the mathematical models and form the development programs of logistics systems in the electric power industry so as to ensure electric power security is based on quite general characteristics of the problem that is being solved. In this regard, the paper can serve as a theoretical basis for working out specific methods to substantiate various development programs of logistics systems, taking into account the characteristics of individual electric power companies and industrial cooperation they are involved in.

\section{Conclusions}

In general, the paper develops a methodological approach to solving the problem of creation of the development program of the logistics system in the electric power industry ensuring electric power security. In this case, we propose a generalized structure of the 
model that can be used to generate the best variant of the development program of the logistics system in the electric power industry with due regard of the limited resources and uncertainty of the influencing factors. In the model we use the requirement for cutting the financial costs during implementation of the program as the criterion of optimality. The uncertainty is accounted for by setting the intervals of possible costs in the implementation of individual projects of the program. In order to resolve the problem we propose an iterative algorithm to successively form the optimal variant of the program.

\section{References}

1. D.A. Melnik, A.Y. Chernysh, Electric power security as a system element of energy and economic security in the system of national security of the Russian Federation (Moscow, Sovremennaya nauchnaya mysl', 2017)

2. I.M. Zaychenko et al., E3S Web of Conferences 110, 02041 (2019) DOI https://doi.org/10.1051/e3sconf/201911002041

3. T. Saurenko et al., E3S Web of Conferences 217, 07025 (2020) DOI: https://doi.org/10.1051/e3sconf/202021707025.

4. N. Grashchenko et al., International Scientific Conference Energy Management of Municipal Facilities and Sustainable Energy Technologies EMMFT (2018) DOI: 9783-030-19868-8_30

5. E. Zotova, et al., E3S Web of Conferences 110, 02053 (2019)

6. E.S. Rodionova, et al., International Journal of Supply Chain Management 8(1), 86-89 (2019)

7. M.A. Sonkin et al., J. Phys.: Conf. Ser. 803(1), 012006 (2017) DOI: 10.1088/1742$6596 / 803 / 1 / 012006$

8. O. Yastrebov et al., SHS Web of Conf. 44, 00009 (2018) DOI: https://doi.org/10.1051/shsconf/20184400009

9. I.V. Ilin, V.I. Koposov, A.I. Levina, Life Science Journal 11(11), 265-269 (2014)

10. A.F. Suprun, et al., Automatic Control and Computer Sciences 52(8), 1105-1110 (2018) DOI: $10.3103 / \mathrm{S} 0146411618080035$

11. A. Chernysh, et al., E3S Web of Conferences $\mathbf{0 3 0 0 3}$ (2018) DOI: https://doi.org/10.1051/e3sconf/20183303003

12. M.A. Sonkin, et al., International Journal of Scientific and Technology Research 9(2), 6259-6262 (2020)

13. A.H. Land, A.G. Doig, Econometrica 28, 497-520 (1960)

14. M.A. Sonkin et al., Proceedings of the 2016 Conference on Information Technologies in Science, Management, Social Sphere and Medicine (ITSMSSM 2016), 282-285 (2016) DOI https://doi.org/10.2991/itsmssm-16.2016.57

15. V.G. Anisimov, Ye.G. Anisimov, USSR Computational Mathematics and Mathematical Physics. 29(5), 238 - 241 (1989)

16. A.O. Alekseyev, et al., USSR Computational Mathematics and Mathematical Physics 25(6), 50-54 (1985)

17. V.P. Los et al., Automatic Control and Computer Sciences 54(8), 930-936 (2020) DOI: $10.3103 / \mathrm{S} 0146411620080374$

18. G. Silkina, IOP Conference Series: Materials Science and Engineering (2019) https://doi.org/10.1088/1757-899X/497/1/012018 
19. T. Saurenko et al., E3S Web of Conferences 217, 07024 (2020) DOI: https://doi.org/10.1051/e3sconf/202021707024

20. E.A. Zotova, et al., Information and Control Systems 5, 18-23 (2019) DOI: 10.31799/1684-8853-2019-5-18-23

21. I.V. Ilin et al., Proceedings of 2017 20th IEEE International Conference on Soft Computing and Measurements, SCM 2017 7970732, 812-814 (2017) DOI: 10.1109/SCM.2017.7970732.

22. A. Levina, et al., MATEC Web of Conferences 170, 01038 (2018) DOI: https://doi.org/10.1051/matecconf/201817001038

23. I. Bagaeva, et al., E3S Web of Conferences, St. Petersburg 02066 (2019) DOI: https://doi.org/10.1051/e3sconf/201911002066

24. D.A. Bazhin et al., Automatic Control and Computer Sciences 50(8), 717-721 (2016) DOI: $10.3103 / \mathrm{S} 0146411616080289$

25. S.P. Prisyazhnyuk, et al., Automatic Control and Computer Sciences 51(8), 824-828 (2017) DOI: $10.3103 / \mathrm{S} 0146411617080053$

26. M. Sonkin, et al., International Journal of Applied Engineering Research 10(17), 38127-38132 (2015)

27. S. Krasnov, et al., E3S Web of Conferences 110, 02052 (2019) DOI: $10.1051 / \mathrm{e} 3$ sconf $/ 201911002052$.

28. A.O. Alekseyev, et al., Soviet Journal of Computer and Systems Sciences 5, 130 - 134 (1988)

29. V.G. Anisimov, Ye.G. Anisimov, USSR Computational Mathematics and Mathematical Physics 32(12), 1827-1832 (1992)

30. P.D. Zegzhda et al., Automatic Control and Computer Sciences 53(8), 1038-1044 (2019) DOI: $10.3103 / \mathrm{S} 0146411619080054$ 